\title{
Application of Accelerometer Data to Mars Odyssey Aerobraking and Atmospheric Modeling
}

\author{
R. H. Tolson ${ }^{1}$, G. M. Keating ${ }^{2}$, B. E. George ${ }^{3}$, P. E. Escalera ${ }^{3}$, and M. R. Werner ${ }^{3}$ \\ George Washington University, Joint Institute for the Advancement of Flight Sciences \\ and \\ A. M. Dwyer ${ }^{4}$ and J. L. Hanna ${ }^{4}$ \\ NASA Langley Research Center \\ Hampton, VA 23681-2199
}

\begin{abstract}
Aerobraking was an enabling technology for the Mars Odyssey mission even though it involved risk due primarily to the variability of the Mars upper atmosphere. Consequently, numerous analyses based on various data types were performed during operations to reduce these risk and among these data were measurements from spacecraft accelerometers. This paper reports on the use of accelerometer data for determining atmospheric density during Odyssey aerobraking operations. Acceleration was measured along three orthogonal axes, although only data from the component along the axis nominally into the flow was used during operations. For a one second count time, the RMS noise level varied from 0.07 to $0.5 \mathrm{~mm} / \mathrm{s}^{2}$ permitting density recovery to between 0.15 and $1.1 \mathrm{~kg} / \mathrm{km}^{3}$ or about $2 \%$ of the mean density at periapsis during aerobraking. Accelerometer data were analyzed in near real time to provide estimates of density at periapsis, maximum density, density scale height, latitudinal gradient, longitudinal wave variations and location of the polar vortex. Summaries are given of the aerobraking phase of the mission, the accelerometer data analysis methods and operational procedures, some applications to determining thermospheric properties, and some remaining issues on interpretation of the data. Pre-flight estimates of natural variability based on Mars Global Surveyor accelerometer measurements proved reliable in the mid-latitudes, but overestimated the variability inside the polar vortex.
\end{abstract}

\section{Nomenclature}

A aerodynamic reference area

a acceleration

AAG Atmosphere Advisory Group

AMT Atmosphere Modeling Team

$\mathrm{C}_{\mathrm{y}} \quad \mathrm{y}$-axis aerodynamic force coefficient

FDS Flight Data System

GDS Ground Data System

h areodetic altitude

$\mathrm{h}_{\mathrm{o}} \quad$ areodetic reference altitude

$\mathrm{H}_{\mathrm{S}}$ density scale height

LaRC Langley Research Center

LMA Lockheed Martin Aerospace

$\mathrm{L}_{\mathrm{S}} \quad$ celestial longitude of Mars

m Odyssey mass

MGS Mars Global Surveyor

MOI Mars orbit insertion

\footnotetext{
${ }^{1}$ Professor, Associate Fellow

${ }^{2}$ Senior Research Staff Scientist, Associate Fellow

${ }^{3}$ Graduate Research Scholar Assistant

${ }^{4}$ Aerospace Engineer, Vehicle Analysis Branch, ASCAC
}

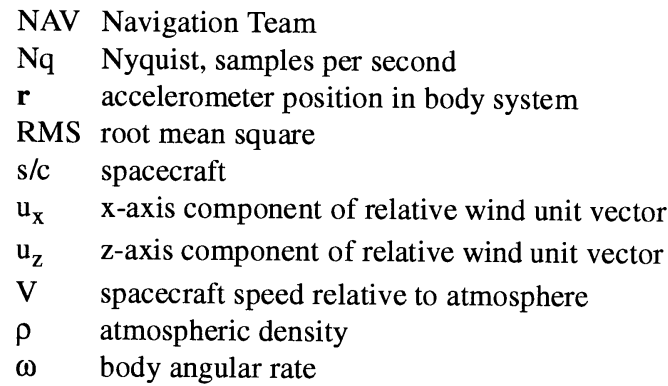

\section{Introduction}

Aerobraking is the utilization of atmospheric drag for beneficial orbit changes via multiple passes through an atmosphere. The first application of aerobraking in a planetary mission was during the Magellan mission at Venus (Ref. 1). To increase imaging radar and gravity field resolution in the polar region, aerobraking was performed during the extended mission in 1993 over about 750 orbital passes to reduce the eccentricity from 0.3 to 0.03 in about 70 days. During Magellan, adjustments were made to the Venus atmospheric model based on orbital decay drag data. The second application 
began in Sept. 1997 when over 850 MGS aerobraking passes were used to reduce the post-MOI period from about 45 hours to about 2 hours, saving an equivalent impulsive $\Delta \mathrm{V}$ of approximately $1200 \mathrm{~m} / \mathrm{s}$ (Ref. 2). MGS was the first planetary mission where aerobraking was essential for mission success. While the Venusian atmosphere demonstrated less than $10 \% 1-\sigma$ orbit to orbit variability in density, the Mars atmosphere demonstrated between $30 \%$ to $40 \%$. During MGS, persistent density waves were found to exist in the equatorial region which could produce nearly a factor of two change in density from trough to peak. Further, a regional dust storm in the southern hemisphere produced over a factor of two increase in density at the periapsis latitude of $60^{\circ} \mathrm{N}$ (Ref. 3).

The primary drag surfaces for Magellan, MGS and Odyssey were the solar arrays. Solar array temperatures were the pre-aerobraking criteria limiting the pace of aerobraking. Because of the damaged solar array, MGS aerobraking was actually limited by maximum dynamic pressure during each pass at about one half of the heating limit (Ref. 2). The large systematic atmospheric variations discovered during MGS and the random variations confirmed by MGS were included in the Odyssey design. Nevertheless, the mission failures during the previous opportunity lead to numerous additional atmospheric modeling activities (Ref. 4), mission simulations (Ref. 5), and thermal analyses (Ref. 6) prior to Odyssey MOI.

\section{Odyssey Aerobraking Mission Summary}

A detailed overview of the aerobraking phase is given elsewhere (Ref. 7) so only a summary is given here. The aerobraking configuration is shown in Fig. 1. Though not shown here, the bus is surrounded by thermal

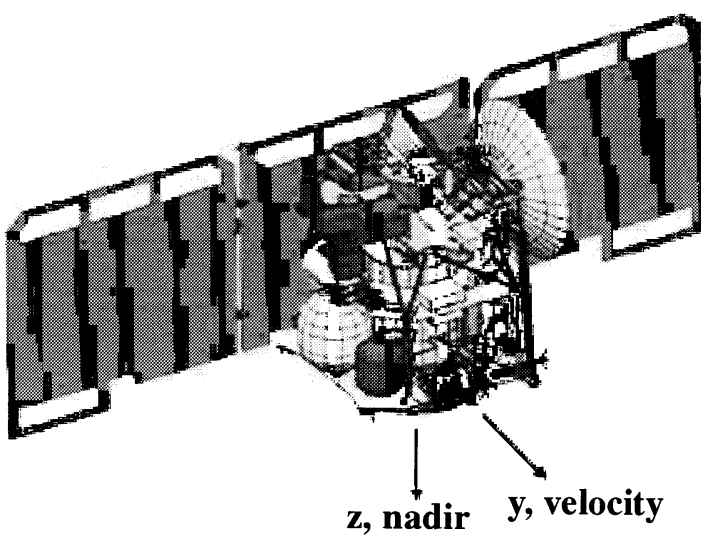

Fig. 1 Mars Odyssey spacecraft in aerobraking configuration, w/o thermal blankets. insulation. This configuration provides strong aerodynamic stability about the body $\mathrm{z}$-axis, which nominally point toward the center of Mars during aerobraking. The s/c is nearly neutrally stable about the $\mathrm{y}$-axis, which is along the velocity direction and normal to the plane of the solar array. The $y$-axis is within $4^{\circ}$ of the aerodynamic trim direction. The photo-voltaic cells are oriented away from the flow to minimize cell heating.

After MOI on October 24, 2001, the orbital period was 18 hours and the goal of aerobraking was to reduce this period to 2 hours by about January 15, 2002. The reference period decay profile, developed just after MOI, is shown in Fig. 2. Also shown is the actual orbital period achieved during aerobraking. The first few orbits were spent checking s/c systems before the periapsis altitude was dropped into the sensible atmosphere. The "walk-in" phase began with orbit 6 with a barely measurable atmospheric effect at $158 \mathrm{~km}$ altitude. Orbit

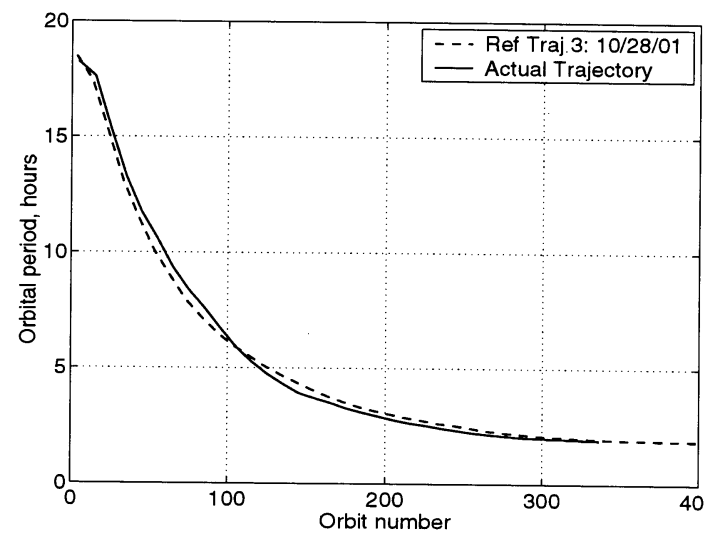

Fig. 2 Planned and actual orbital period during aerobraking.

7 was the first aerobraking pass with a periapsis altitude of $136 \mathrm{~km}$ and a maximum density of about $1.5 \mathrm{~kg} / \mathrm{km}^{3}$. Orbits $17-19$ began the main aerobraking phase with periapsis altitudes between 110.5 and 110.9 and densities between 23 and $40 \mathrm{~kg} / \mathrm{km}^{3}$. The large variations in density anticipated from MGS were clearly present again. The actual orbital decay fell about 50 minutes behind the target at orbit 75 . This was a result of periapsis precessing toward the north pole and passing through the high variability region of the polar vortex. While inside the vortex, the variability was substantially lower and aerobraking could be performed more aggressively, so that by orbit 245 the actual period was 13 minutes ahead of the plan. After 77 days, aerobraking ended on January 11, 2002. This was 13 days shorter than the expected 90 day mission. 
Orbital characteristics of interest for aerobraking are shown in Fig. 3 for the entire aerobraking phase. The figure presents areodetic altitude, latitude, local solar time and $\mathrm{L}_{\mathrm{s}}$ at periapsis. $\mathrm{L}_{\mathrm{s}}$ is the celestial longitude of Mars measured from the Mars vernal equinox and is a measure of Mars season and distance from the Sun. These four variables are thought to be the most significant in determining thermospheric mean properties. The altitude plot shows walk-in (orbits 616), main phase, where solar array temperature was the limiting factor (orbits 17 to 248) and walk-out where orbital lifetime was the limiting factor (orbits 249-336). Precession of the line of apsides due to planetary oblateness $\left(\mathrm{J}_{2}\right)$ moved periapsis poleward for the first 127 orbits and then toward the equator through the end of the mission. Orbit to orbit changes in periapsis
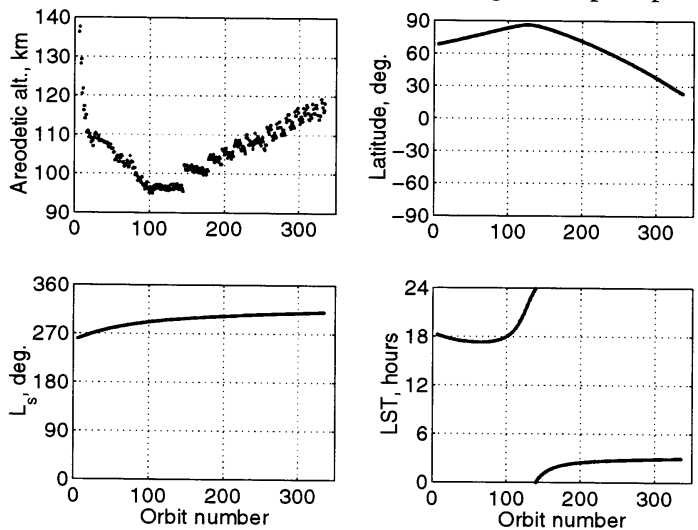

Fig. 3 Variables of interest for aerobraking for the Odyssey mission.

altitude due to short period orbital perturbations are generally less than $1.5 \mathrm{~km}$, so changes larger than this value represent altitude adjustment maneuvers. Geodetic altitude is of interest because in a static atmosphere, equal pressure surfaces would be equipotential surfaces. Up to the time of maximum latitude, the $\mathrm{J}_{3}$ long period variation in eccentricity causes periapsis altitude to increase with time. Likewise, as the periapsis precesses northward due to $\mathrm{J}_{2}$, the flattening of the planet causes areodetic altitude to increase. Both effects reverse after the time of maximum periapsis. These two effects are the same order of magnitude and provided a fail safe situation early in the mission. It is clear that after orbit 140 , these two effects continued to drive periapsis lower into the atmosphere and many maneuvers were performed to keep the altitude in the aerobraking corridor. The general trends are primarily due to the latitudinal density gradient, which at a constant areodetic altitude, causes density to decreases toward the colder pole.
Local solar time, like for all near Hohmann transfers to outer planets, starts near 1800 hours. Since nodal regression is essentially zero for this nearly polar orbit, LST initially becomes earlier due to Mars orbital motion. Eventually northward apsidal precession combined with Mars obliquity begins to dominate and LST moves into night and rapidly shifts to morning hours as periapsis passes over the pole. The aerobraking mission lasted less than two Mars months so the season at Mars remained at northern hemisphere winter $\left(270<\mathrm{L}_{\mathrm{s}}<360\right)$ throughout aerobraking.

\section{Atmospheric Density Recovery}

Since the $y$-component provides the largest acceleration signal to noise ratio (Fig. 1), it was used to recover atmospheric density. The density recovery is based on Newton's second law and the definitions of aerodynamic coefficients

$$
m a_{y}=\frac{\rho v^{2} C_{y} A}{2}
$$

\section{Aerodynamic Data Base}

To use acceleration measurements to determine atmospheric density from Eq. 1, an aerodynamic data base of $\mathrm{C}_{\mathrm{y}}$ is required that covers the $\mathrm{s} / \mathrm{c}$ operational range. Main phase aerobraking was to take place at a nominal heat flux of about $0.3 \mathrm{~W} / \mathrm{cm}^{2}$, which corresponded to an atmospheric density of about $60 \mathrm{~kg} /$ $\mathrm{km}^{3}$ and a Knudsen number of about 0.2 . This Knudsen number is well into the transition region and transitional effects are included by making $\mathrm{C}_{\mathrm{y}}$ a function of the density. Aerodynamic coefficients were generated for eight values of $\rho$ up to at least twice the target density or $120 \mathrm{~kg} / \mathrm{km}^{3}$. Aerodynamic properties were calculated with Direct Simulation Monte Carlo (DSMC) and free molecular flow codes (Ref. 8). The DSMC method was required to accurately quantify aerothermodynamics in the regions of highest dynamic pressure. Preflight attitude control simulations indicated that the relative wind could deviate as much as $20^{\circ}$ from the y-axis. DSMC and free molecular simulations were performed over heading angles up to $28.6^{\circ}$.

The force coefficient over the range of expected densities for flow along the $y$-axis is shown on the left in Fig. 4. The value at $\rho=0.001$ are free molecular flow values and for lower densities this value is used. All calculations are based on assumed momentum accommodation coefficients of unity. The highest periapsis density encountered during aerobraking was 
on orbit 106 with a value of $107.4 \mathrm{~kg} / \mathrm{km}^{3}$. Contours of $\mathrm{C}_{\mathrm{y}}$ variation with wind direction are shown on the right in Fig. 4 for a density of $100 \mathrm{~kg} / \mathrm{km}^{3}$. The variables $u_{x}$ and $\mathrm{u}_{\mathrm{z}}$ are the components of the relative wind unit vector in the s/c body coordinates shown in Fig. 1.
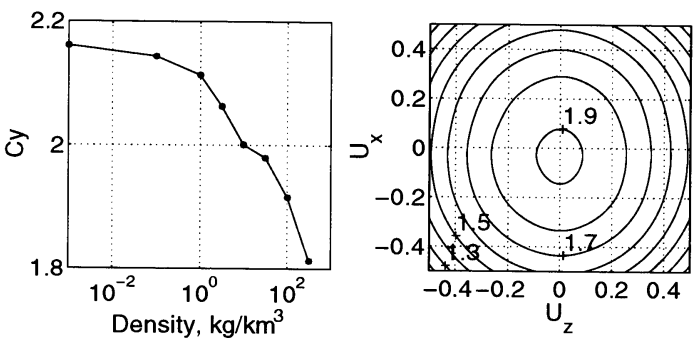

Fig. $4 \mathrm{Y}$-axis force coefficient over a range of atmospheric density for $u_{x}=u_{z}=0$ and versus relative wind direction for $\rho=100 \mathrm{~kg} / \mathrm{km}^{3}$

In recovering density from accelerometer data, the aerodynamic database is used in an iterative manner to solve Eq. 1. For each accelerometer measurement, the relative wind vector, including rigid rotation of the atmosphere with the planet, is determined from the attitude quaternions and the orbital ephemeris. Interpolation into the free molecular versions of Fig. 4 is used to estimate $\mathrm{C}_{\mathrm{y}}$ and thence density. This density is used to update the $\mathrm{C}_{\mathrm{y}}$ estimate and the process continues until density converges to within $1 \%$.

\section{Accelerometer Data}

Two IMU's are located on the upper deck of the s/c as shown in Fig. 1. The primary IMU was used throughout aerobraking. The internal orientation of two of the three accelerometers were not aligned with the body axes, so data from these two were combined in the GDS to form body axes accelerations. The principle acceleration used in the aerobraking analysis is the y direction. The accelerometers are located at $\mathbf{r}=(0.164,-0.5441 .137) \mathrm{m}$ relative to the center of mass. The accelerometers and gyros were sampled at $200 \mathrm{Nq}$. The accelerometer data were quantized internally at $2.7 \mathrm{~mm} / \mathrm{s}$ but the least significant bit in the A/D converter was $0.0758 \mathrm{~mm} / \mathrm{s}$. For the purposes of density recovery, the high rate data were averaged over one second intervals. Up through orbit 136 all data recorded on the s/c at $200 \mathrm{Nq}$ during an aerobraking pass was transmitted to the ground. However, as the eccentricity of the orbit decreased, the duration of the aerobraking pass increased and the full data set could not be recorded onboard. From orbits 137 to orbit 268 , the first 50 samples were recorded for transmission and averaging each second. After orbit
268 , only the first 20 samples or $10 \%$ of the data were transmitted each second. The measured acceleration is composed of a number of terms given by

$$
\mathbf{a}_{\mathrm{m}}=\mathbf{a}_{\mathrm{b}}+\mathbf{a}_{\mathrm{a}}+\mathbf{a}_{\mathrm{g}}+\mathbf{a}_{\mathrm{ACS}}+\omega \times(\omega \times \mathbf{r})+\omega \times \mathbf{r}
$$

where the terms are respectively acceleration due to the instrument bias, aerodynamic forces, gravity gradient (negligible), attitude control system thruster activity, and angular motion of the accelerometer about the center of mass ( 2 terms).

To illustrate the density recovery process, orbit 76 was selected. This orbit provides acceleration variations somewhat similar to the classical "bell curve" and also demonstrates some of the local variations during a pass. The raw accelerometer data along the $y$-axis from which density was derived is shown in Fig. 5 . Note that the onboard process for removing bias has left a small residual. This residual bias is removed, as discussed below, before density is derived from the data. Also as described above, in the derivation of density, the process simultaneously recovers the $\mathrm{C}_{\mathrm{y}}$ coefficient. The value of $\mathrm{C}_{\mathrm{y}}$ for this pass is shown in the upper left. The $\mathrm{C}_{\mathrm{y}}$ value decreases about $10 \%$ during the 150 seconds before periapsis primarily due to the transition flow phenomena shown in Fig. 4. On the outbound portion of the pass, $\mathrm{C}_{\mathrm{y}}$ returns to the free molecular value just prior to 200 seconds and then decreases due, as will be seen later, to a change in heading which exposes less cross sectional area to the flow.

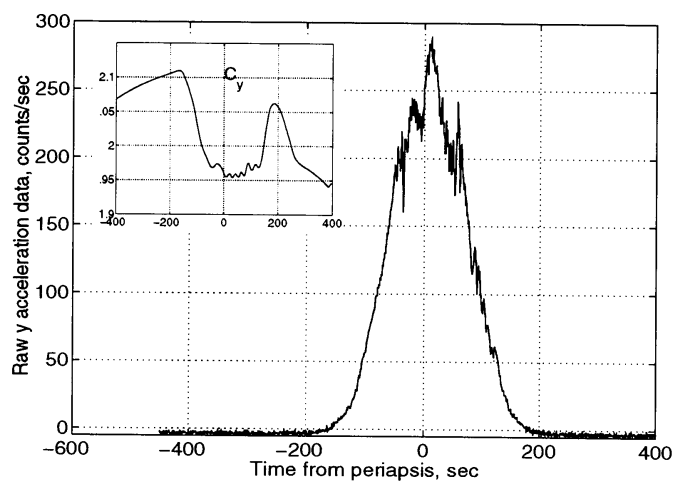

Fig. 5 Raw accelerometer counts and calculated axial aerodynamic force coefficient, $P 076$.

Accelerometer bias was calibrated on the s/c by averaging measurements prior to encountering the atmospheric and after leaving the atmosphere. This constant value was automatically subtracted from the measurements in the FDS before the one second averaging was done. Subsequent analysis showed that 
the bias was different between the inbound and outbound legs of a pass, probably due to the general increase in temperature of the IMU throughout a pass. During AMT operations, a linear time dependent bias was determined using inbound and outbound data. Through the pass, this model was evaluated at each observation time. An example of the process is shown in Fig. 6. The upper plot shows the 1 second averaged
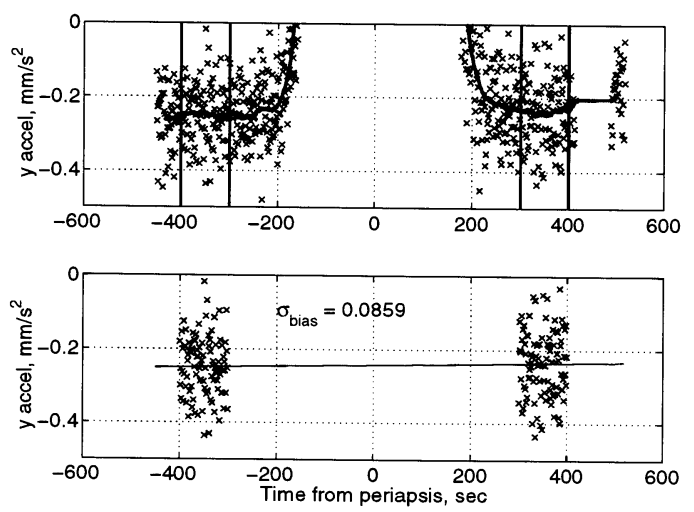

Fig. 6 Accelerometer bias removal process for P076.

acceleration data from the GDS. The line is the seven point running mean. To determine the bias, inbound and outbound data were selected visually, as indicated by the vertical lines, and a least square fit is use to obtain the linear model. The results are shown in the lower part of the figure. Though small compared to the RMS residual of $0.086 \mathrm{~mm} / \mathrm{s}$, the trend in bias is clearly present and the value is typical of most orbits. Note that the noise level of $0.086 \mathrm{~mm} / \mathrm{s}$ is fairly close to the least significant bit value of $0.0758 \mathrm{~mm} / \mathrm{s}$. The noise level, determined during the bias calculation, for each orbit is shown in
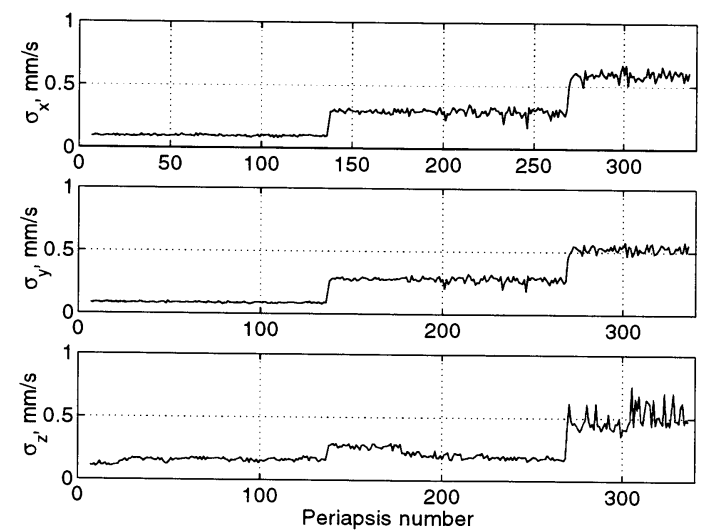

Fig. 7 Variation in accelerometer noise level throughout the mission.
Fig. 7. The influence of changing the number of high rate samples from 200, to 50 and then to 20 is clearly evident. The lowest noise level corresponds to determining density to about $0.15 \mathrm{~kg} / \mathrm{km}^{3}$ and the largest to about $1.1 \mathrm{~kg} / \mathrm{km}^{3}$.

\section{Other Data Types}

Angular motion contributions to the acceleration (Eq. 2) were removed using the rate gyro data that were received at $1 \mathrm{Nq}$ also. A typical history of the body rates is shown in Fig. 8. Recall that rotations about the y-axis

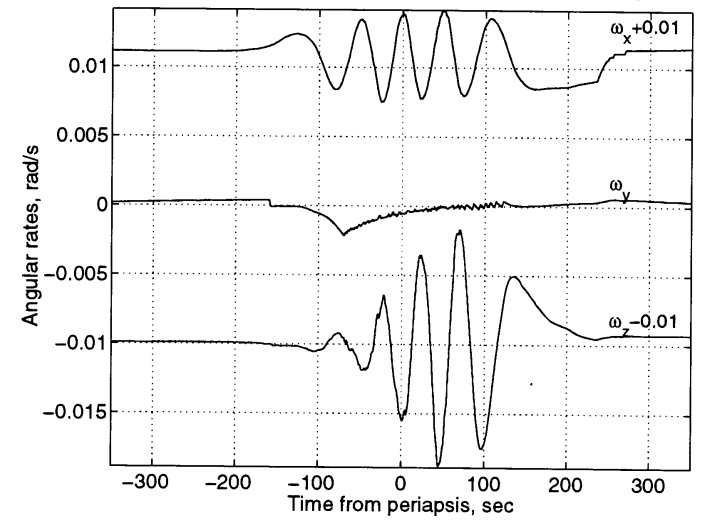

Fig. 8 Body angular rates during P076, rates displaced for clarity

(Fig. 1) have essentially no aerodynamic restoring force, but motion about all three axes are coupled through the on board momentum supplied by the reaction wheels. From the $y$-axis angular rate data it is seen that a large thruster firing took place at about -150 seconds and then nearly continuous firings from -75 seconds through about 120 seconds. These particular firings are coupled and theoretically produce no net acceleration of the s/c. The angular acceleration required in Eq. 2 was determined by fitting a polynomial to the rates and then differentiating the polynomial to determine the acceleration at the central point. For typical aerobraking passes the maximum contribution due to these two terms is less than $0.5 \mathrm{~mm} / \mathrm{s}^{2}$, which, though small, is sufficiently large to require inclusion.

The orientation of the relative wind is obtained from the orbital ephemeris and the quaternions, also averaged to $1 \mathrm{Nq}$. The history of the relative wind is shown in Fig. 9 for orbit 76. From this figure and Fig. 8 it is seen that aerodynamic torques are significant within about 150 seconds of periapsis and the aerodynamic stability about the $\mathrm{x}$ and $\mathrm{z}$ axes is evident during these times. While in the atmosphere, deviations in $u_{x}$ and $u_{z}$ do not exceed 0.1 or less than $6^{\circ}$. The large outbound excursion is due 
to loss of aerodynamic stability on exiting the atmosphere, so the s/c continues to rotate until the attitude control system becomes active. This excursion is the reason for the outbound decrease in $\mathrm{C}_{\mathrm{y}}$ shown in Fig. 5. Note that the center of oscillation is near $u_{z}$ of 0.07 corresponding to the equilibrium pitch angle of about $4^{\circ}$. This offset is primarily due to the geometric asymmetry caused by the high gain antenna (Fig. 1).

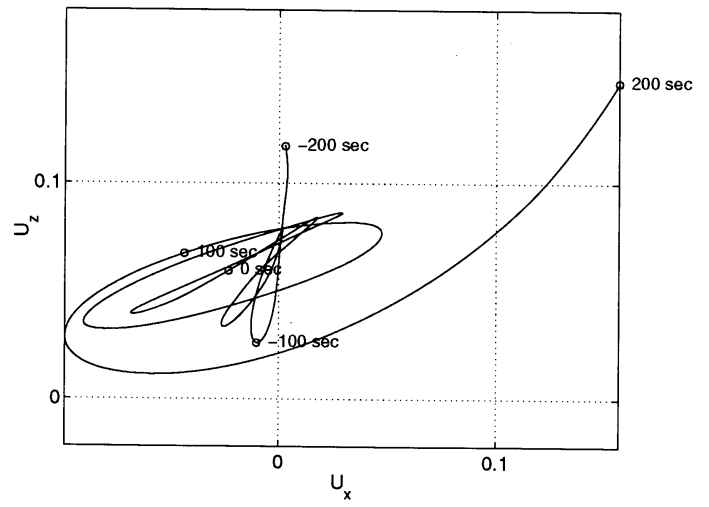

Fig. 9 Relative wind orientation during P076, times are seconds from periapsis.

Acceleration caused by thruster firing is the most difficult to remove. The factors that determine thruster effectiveness include specific impulse, propellant blow down, temperature of the catalyst bed, and interference with the flow (Ref. 9). Past experience has shown that calibration within $50 \%$ is difficult for the short thrusting times and variable duty cycle typically associated with aerobraking attitude control (Ref. 10). The Odyssey thrusters were calibrated during interplanetary cruise (Ref. 11), but the calibration was found to be unreliable for the orbital phase. Since the contribution to the total acceleration is two orders of magnitude less than the periapsis drag effect, an ad hoc correction was made during each pass by the NAV (Ref. 12). Due to the smallness of the correction, accelerometer data during operations was processed using the original interplanetary calibration. Post flight analysis of the data to extend the applicability to higher altitudes will require an improved calibration much like that performed for MGS data (Ref. 13).

\section{Operational Procedures}

There were three activities determining atmospheric models during operations. The Odyssey navigation team (NAV), the Atmospheric Modeling Team (AMT), and the Atmospheric Advisory Group (AAG). The NAV team utilized radio tracking data to determine the drag effect for each orbit (Ref. 7). The AMT utilized orbit determination products from NAV and accelerometer and other telemetry data to determine density every second throughout each aerobraking pass and to produce products for NAV, other LaRC teams, (Ref. 6, Ref. 9) and the AAG. AAG members were atmospheric scientist who reviewed and interpreted all available data and made recommendations to the project flight manager on periapsis altitude control maneuvers planned for the next maneuver opportunity.

Because there were no tracking data during aerobraking, radio tracking can essentially only determine the effective $\Delta \mathrm{V}$ associated with the total drag pass. To map this into equivalent atmospheric parameters, MarsGRAM (Ref. 14) was used as the underlying model for the time dependence of density during the pass and NAV solved for a density multiplier that provided the best fit to pre- and post-aerobraking tracking data for each orbit (Ref. 7). NAV utilized a density dependent drag coefficient similar to Fig. 4 but neglected changes in drag due to $\mathrm{s} / \mathrm{c}$ orientation also shown in Fig. 4.

The operations plan called for NAV to process radio tracking data prior to the beginning of the drag pass and provide predictions of the osculating elements at the subsequent periapses. These predictions were called "preliminary orbits." A "final" orbit meant that both pre- and post-aerobraking radio tracking data had been used in the orbit determination. Final orbit determinations were typically available from NAV about 2 hours after periapsis. The AMT was located at Langley Research Center (LaRC) on the east coast. Operations typically began at 0700 hours eastern with transferring the previous days data from the GDS to a LaRC server. The AMT team used final orbits, when available, to process accelerometer data accumulated overnight to determine periapsis density, maximum density, density scale height in the vicinity of periapsis, latitudinal gradient of both density and scale height, density and scale height at reference altitudes of 100 , $110 \ldots 200 \mathrm{~km}$, and other atmospheric variables. AMT also determined a MarsGRAM density multiplier directly from accelerometer data for comparison with the NAV value. These results were transmitted to a file server in flight operations for NAV and AAG review. At 1130 pacific, the AAG met to discuss all atmospheric results and develop a maneuver recommendation and rationale. The maneuver options were "no maneuver", "up maneuver" (i.e. raise periapsis altitude by some number of kilometers) or "down maneuver" (i.e. lower periapsis altitude by some number of kilometers). At 1430 pacific, AAG, NAV and LMA s/c system team shared their recommendations with the Flight 
Operations Manager for a final decision on the maneuver to be performed at the next opportunity.

\section{Results}

The first result developed by the AMT was the variation of density with time for each pass. These data were supplied to other teams to perform thermal analyses, flight dynamics simulations, and other studies. From the density vs. time data, numerous parameters as mentioned above were extracted for AMT, AAG and NAV use. Discussed below are selected results on density vs. time and density-altitude profiles. Utilization of the orbit to orbit variations are then discussed in terms of prediction methods used for Odyssey. Finally the method used to locate the polar vortex is presented.

\section{P076 Density Profiles}

Three realizations of density for P076 are shown in Fig. 10. The lower curve is the density every second, the middle curve is the 7 point average and the upper curve is the 39 point average. The curves are displaced for clarity. The 7 point averaging is done to remove local spatial variations in density but leave "mesoscale" wave structure in the $100 \mathrm{~km}$ wavelength category. Some of these waves will be discussed later in the section on the polar vortex. The 39 point averaged data are used to estimate the "mean" atmosphere. The latter data were used to estimate density and density scale height at periapsis, latitudinal temperature and density gradients, exospheric temperatures, and identify inbound and outbound properties for operational prediction.

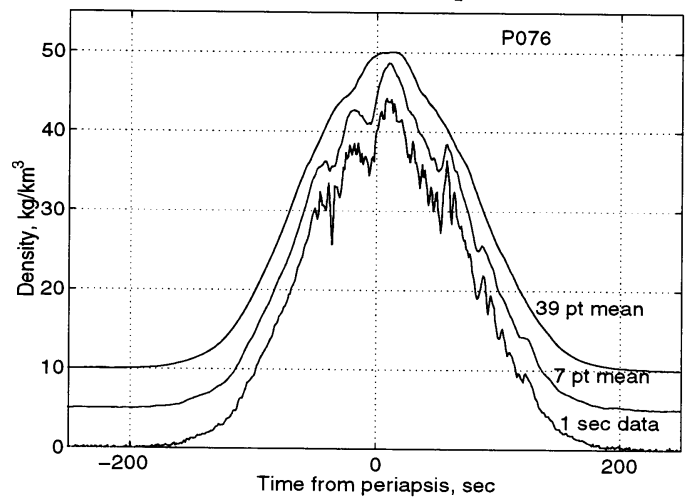

Fig. 10 Raw and smoothed derived density for P076

The rapid changes in density around $-40 \mathrm{sec}$. and +50 sec. are real variations in the atmosphere. Like these occurrences, it is not unusual for density to change by $30 \%$ in a few seconds. At 50 seconds from periapsis the $\mathrm{s} / \mathrm{c}$ down track speed is about $4.5 \mathrm{~km} / \mathrm{s}$ and the radial component is about $120 \mathrm{~m} / \mathrm{s}$. As with MGS (Ref. 3), small spatial scale variations of this order occur on a majority of passes. On at least one MGS case (Ref. 13), there is a compelling argument that density changed by a factor of 5 in less than 5 seconds during which the s/c traveled about $1.3 \mathrm{~km}$ lower in altitude and $20 \mathrm{~km}$ down track. Returning to Fig. 10, the 7 point averaged profile suggest between 3 and 5 mesoscale waves about 20 seconds apart or about $90 \mathrm{~km}$ if these are purely latitudinal structures. Additional Odyssey passes will be presented later to demonstrate a variety of profiles.

To relate density vs. time to atmospheric properties of interest to operations, a number of assumptions, simplifications and considerations must be noted. First, Odyssey is in a near polar orbit and over a typical aerobraking pass the $\mathrm{s} / \mathrm{c}$ is in the detectable atmosphere less than 400 seconds. While in the atmosphere, the latitude varies between $\sim 20^{\circ}$ at the beginning to $\sim 50^{\circ}$ at the end of the mission. The s/c travels between $12^{\circ}$ and $26^{\circ}$ in latitude while within one density scale height $(\sim 7$ $\mathrm{km}$ ) of periapsis. Thus, latitudinal variations cannot be ignored in the Odyssey profiles and the common assumption of hydrostatic equilibrium is probably not applicable across an entire pass. With the 1 second data, density actually increase with altitude for many orbits and it is not uncommon with the 7 second averaged data. Such variations suggest that the atmosphere is not in static equilibrium over even small scales.

The models most utilized during operations included (1) the constant density scale height $\left(\mathrm{H}_{\mathrm{s}}\right)$ model usually applied to a limited altitude range in the vicinity of a reference altitude $\left(\mathrm{h}_{\mathrm{o}}\right)$ on the inbound leg, the outbound leg, or near periapsis.

$$
\rho(h)=\rho\left(h_{0}\right) e^{\left[\frac{-\left(h-h_{0}\right)}{H_{s}}\right]}
$$

(2) the model with constant density scale height but density at the reference altitude $\rho\left(\mathrm{h}_{\mathrm{o}}\right)$ varying linearly with latitude, and (3) the model with both reference altitude density and density scale height varying linearly with latitude. Under the assumptions of hydrostatic equilibrium and isothermal atmosphere, density scale height is directly proportional to temperature. The last model is thus approximately equivalent to assuming that density and temperature at a reference altitude vary linearly with latitude. Deviation of the atmosphere from hydrostatic equilibrium and constant temperature will bias temperature derived using this connection. Nevertheless, the few temperatures mentioned in this paper are derived under these assumptions.

The altitudinal profile for P076 is shown in Fig. 11. Within about $10 \mathrm{~km}$ of periapsis there is little difference 
between the density or density scale height for the inbound and outbound legs. Between 110 and $160 \mathrm{~km}$ altitude, the inbound leg, which is north of periapsis, appears to have a much lower temperature than the outbound leg. This is not unexpected since the outbound leg is at a lower latitude and moving toward what should be the warmer equator. At $140 \mathrm{~km}$ the local density scale heights are $6.40 \mathrm{~km}$ inbound and $8.84 \mathrm{~km}$ outbound. Interpreting these scale heights in terms of a locally isothermal atmosphere yields temperatures of $114 \mathrm{~K}$ and $157 \mathrm{~K}$, respectively.

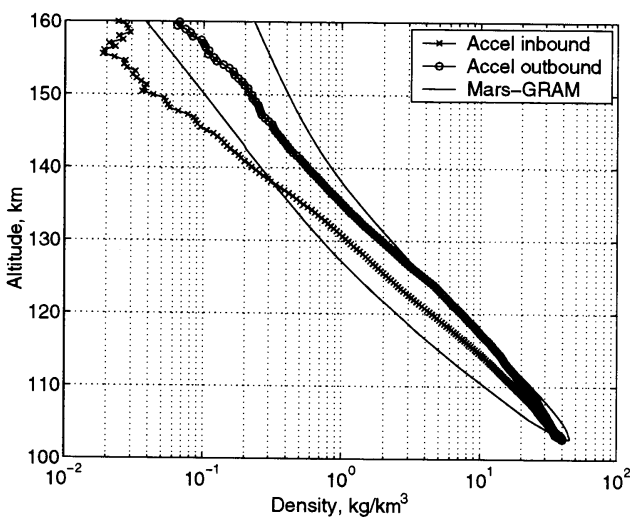

Fig. 11 Derived density versus altitude for P076 compared with scaled MarsGRAM profile.

The profile from MarsGRAM is also included on the figure. MarsGRAM densities have been multiplied by 0.665 to provide the same effective total $\Delta \mathrm{V}$ for the pass, that is, the same area under the density vs. time curve. Near periapsis, the MarsGRAM model shows a much stronger latitudinal gradient than inferred from the accelerometer data. The difference becomes larger with altitude with MarsGRAM predicting a factor of 3.5 density ratio between inbound and outbound at $140 \mathrm{~km}$ while only a factor of 2.5 was measured. From an overall mission viewpoint this would be considered a "good" comparison.

Fig. 12 shows the periapsis density and density scale height for each orbit during aerobraking. These results were derived by performing a least squares fit to the log density profile using all data within $10 \mathrm{~km}$ of the periapsis altitude to determine $\rho\left(h_{0}\right)$ and $H_{s}$ in Eq. 3 . The periapsis density variation shows the main aerobraking phase up to about orbit 250 where solar array temperature is the controlling factor. This phase is followed by the walk out phase where orbit lifetime is the major consideration. Note from Fig. 3 that periapsis altitude is smoothly decreasing up to orbit 100 , yet the periapsis density only slightly reflects this trend and up to orbit 75 the orbit to orbit variations can be up to a factor of 4 . Orbits 100 through 150 are at about the same altitude and the orbit to orbit variability is much smaller than the earlier orbits or later orbits. During this time periapsis is above $80^{\circ}$ latitude and the lack of variability is interpreted as being inside the polar vortex. on which there will be more later.

During the first 100 orbits, density scale height (temperature) increases as periapsis precesses toward the pole and altitude decreases (Fig. 3). This is an unexpected result since both trends were expected to result in a decrease in temperature. This anomalistic trend has been interpreted (Ref. 15) as a winter polar mesospheric warming that raised the entire temperature structure of the upper atmosphere in the polar region. Such a phenomena was not predicted based on the MarsGRAM model and may not be an annual event.
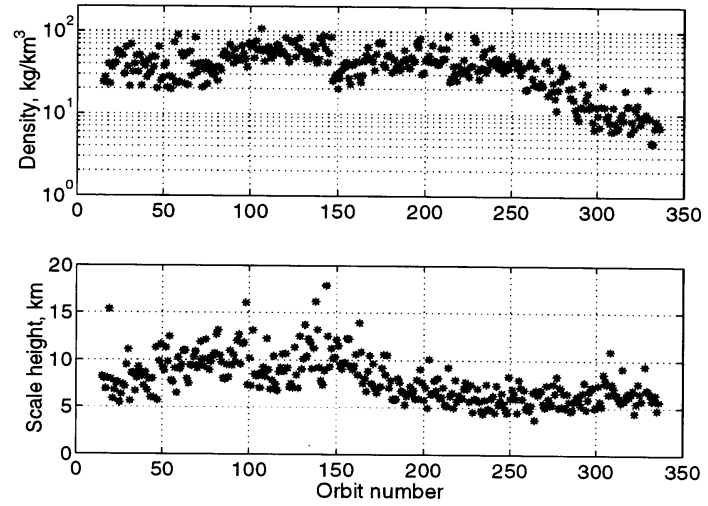

Fig. 12 Periapsis density and density scale height.

\section{Other Density Profiles}

As was experienced during MGS, there are a number of interesting phenomena that have occurred in the exploration of the thermosphere of Mars during aerobraking. Examples of these are shown in Fig. 13. The equivalent MarsGRAM profile is also plotted for comparison. The outbound leg of orbit 155 shows a traditional bell shaped variation with time closely following MarsGRAM. Periapsis occurs at about $82^{\circ} \mathrm{N}$ latitude and $270^{\circ} \mathrm{E}$ longitude. The outbound leg is poleward of periapsis. The "bump" in the inbound leg at $-100 \mathrm{sec}$ is about twice the equivalent outbound density and occurs at about $75^{\circ}$ latitude. These types of profiles were interpreted as crossing the polar vortex. There is highly variable wave like structures outside and near the vortex and relatively smooth variations inside the vortex. The polar vortex is similar to the northern hemisphere jet stream that is highly variable in both time and space but generally rotates with the planet while migrating eastward. Orbit 157 has nearly the same 
between the density or density scale height for the inbound and outbound legs. Between 110 and $160 \mathrm{~km}$ altitude, the inbound leg, which is north of periapsis, appears to have a much lower temperature than the outbound leg. This is not unexpected since the outbound leg is at a lower latitude and moving toward what should be the warmer equator. At $140 \mathrm{~km}$ the local density scale heights are $6.40 \mathrm{~km}$ inbound and $8.84 \mathrm{~km}$ outbound. Interpreting these scale heights in terms of a locally isothermal atmosphere yields temperatures of $114 \mathrm{~K}$ and $157 \mathrm{~K}$, respectively.

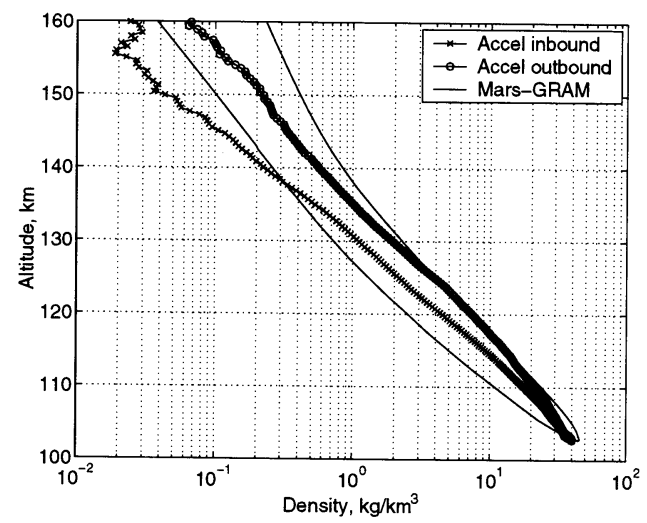

Fig. 11 Derived density versus altitude for $\mathrm{P076}$ compared with scaled MarsGRAM profile.

The profile from MarsGRAM is also included on the figure. MarsGRAM densities have been multiplied by 0.665 to provide the same effective total $\Delta \mathrm{V}$ for the pass, that is, the same area under the density vs. time curve. Near periapsis, the MarsGRAM model shows a much stronger latitudinal gradient than inferred from the accelerometer data. The difference becomes larger with altitude with MarsGRAM predicting a factor of 3.5 density ratio between inbound and outbound at $140 \mathrm{~km}$ while only a factor of 2.5 was measured. From an overall mission viewpoint this would be considered a "good" comparison.

Fig. 12 shows the periapsis density and density scale height for each orbit during aerobraking. These results were derived by performing a least squares fit to the $\log$ density profile using all data within $10 \mathrm{~km}$ of the periapsis altitude to determine $\rho\left(h_{0}\right)$ and $H_{s}$ in Eq. 3 . The periapsis density variation shows the main aerobraking phase up to about orbit 250 where solar array temperature is the controlling factor. This phase is followed by the walk out phase where orbit lifetime is the major consideration. Note from Fig. 3 that periapsis altitude is smoothly decreasing up to orbit 100 , yet the periapsis density only slightly reflects this trend and up to orbit 75 the orbit to orbit variations can be up to a factor of 4 . Orbits 100 through 150 are at about the same altitude and the orbit to orbit variability is much smaller than the earlier orbits or later orbits. During this time periapsis is above $80^{\circ}$ latitude and the lack of variability is interpreted as being inside the polar vortex. on which there will be more later.

During the first 100 orbits, density scale height (temperature) increases as periapsis precesses toward the pole and altitude decreases (Fig. 3). This is an unexpected result since both trends were expected to result in a decrease in temperature. This anomalistic trend has been interpreted (Ref. 15) as a winter polar mesospheric warming that raised the entire temperature structure of the upper atmosphere in the polar region. Such a phenomena was not predicted based on the MarsGRAM model and may not be an annual event.
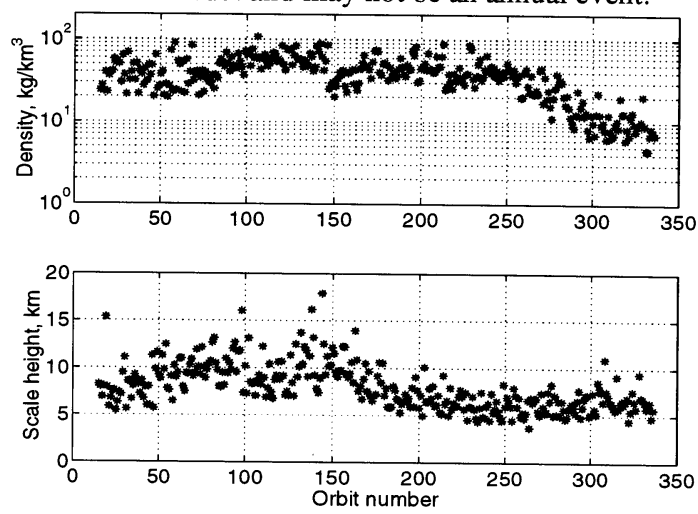

Fig. 12 Periapsis density and density scale height.

\section{Other Density Profiles}

As was experienced during MGS, there are a number of interesting phenomena that have occurred in the exploration of the thermosphere of Mars during aerobraking. Examples of these are shown in Fig. 13. The equivalent MarsGRAM profile is also plotted for comparison. The outbound leg of orbit 155 shows a traditional bell shaped variation with time closely following MarsGRAM. Periapsis occurs at about $82^{\circ} \mathrm{N}$ latitude and $270^{\circ} \mathrm{E}$ longitude. The outbound leg is poleward of periapsis. The "bump" in the inbound leg at $-100 \mathrm{sec}$ is about twice the equivalent outbound density and occurs at about $75^{\circ}$ latitude. These types of profiles were interpreted as crossing the polar vortex. There is highly variable wave like structures outside and near the vortex and relatively smooth variations inside the vortex. The polar vortex is similar to the northern hemisphere jet stream that is highly variable in both time and space but generally rotates with the planet while migrating eastward. Orbit 157 has nearly the same 
ground track as orbit 155 but with periapsis at $162^{\circ} \mathrm{E}$ longitude. The interpretation here is that periapsis is outside the vortex in this longitude range and latitudinal waves are increasing the density variations at both -100 sec. and $+75 \mathrm{sec}$ while decreasing it near periapsis, resulting in the plateau shown.
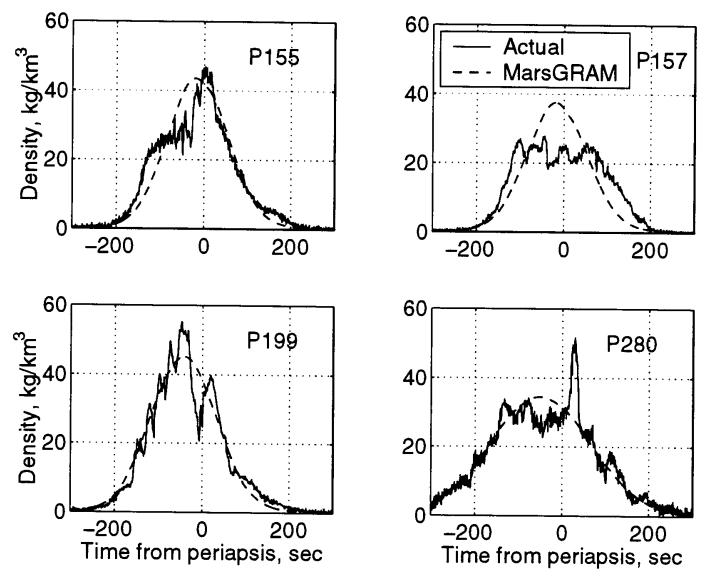

Fig. 13 Other atmospheric density profiles.

By orbit 199 periapsis has precessed to $72^{\circ} \mathrm{N}$. The inbound leg has 5 waves up to the maximum density. For these waves, the peak to trough density ratios vary between 1.2 and 1.9 and the peaks are about $2^{\circ}$ apart in latitude. The last peak just after periapsis is followed by a very low variability outbound leg after $75 \mathrm{sec}$. at a latitude of $78^{\circ}$ The interpretation is that the vortex boundary is near $76^{\circ}$ giving a highly variable profile up to $75 \mathrm{sec}$. while outside the vortex and low variability inside the vortex after $75 \mathrm{sec}$. Finally, orbit 280 is included to show that local phenomena can produce nearly factor of 2 changes in density over very short time scales. The latitude range goes from $25^{\circ} \mathrm{N}$ at -200 $\sec$ to $63^{\circ} \mathrm{N}$ at $+200 \mathrm{sec}$. The spike just after periapsis has a latitude width of about $2^{\circ}$. The Mars thermosphere is noted for such large spatial and temporal variability. For an aerobraking mission it should be kept in mind that the solar array temperature is the limiting factor and that conduction through the solar array smooths many of these short term variations and such local peaks may contribute little to the maximum temperature (Ref. 6).

\section{Using Accelerometer Data for Prediction}

As seen from the previous section, the thermosphere of Mars at aerobraking altitudes is highly variable. This section will discuss the various methods that used accelerometer data for predicting density for future orbits. Essentially all of the methods were used each day and evaluated and compared.

\section{Persistence and MarsGRAM Scaling}

The simplest prediction method is persistence, that is, assume the density profile for the next orbit will be the same as the last orbit. Since the altitudes may be different, periapsis density from the last orbit $(\rho(n))$ is mapped to the periapsis altitude $(h(n+1))$ of the next orbit via the density scale height $\left(\mathrm{H}_{\mathrm{s}}(\mathrm{n})\right)$ using Eq. 3 to yield the estimate

$$
\hat{\rho}(n+1)=\rho(n) e^{\left[\frac{h(n)-h(n+1)}{H_{s}(n)}\right]} .
$$

Using the density and scale heights in Fig. 12 and the altitudes from Fig. 3, the ratios of actual for the next orbit to the predicted for the next orbit are shown inFig. 14. The mean ratio is 1.10 and the standard deviation over the entire phase is 0.49 . The biased estimate is due to the overweighting of a large ratio compared to the reciprocal of a large ratio. The orbit to orbit variability is clearly larger during the first 75 and last 150 orbits. The
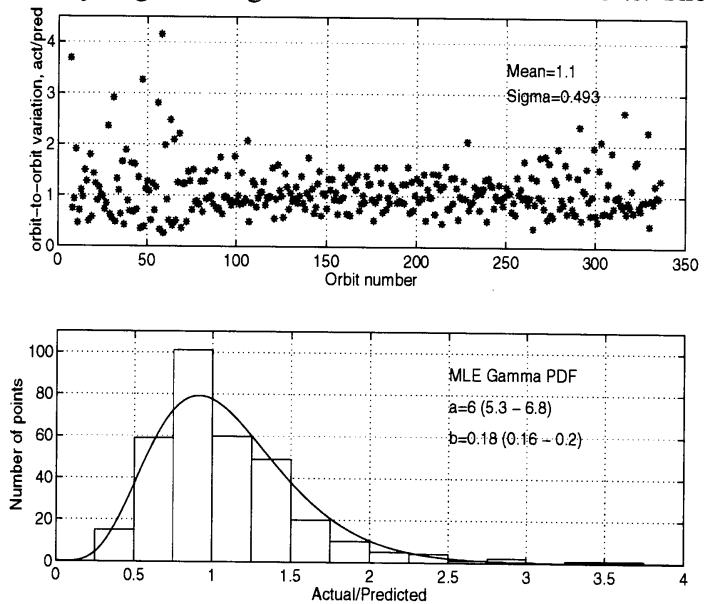

Fig. 14 Prediction capability for persistence throughout aerobraking.

early high variability is associated with waves near the polar vortex. After periapsis has precessed into the polar region and moved into the nighttime (Fig. 3), variability decreases. As periapsis precesses toward the equatorial region, near the end of aerobraking, variability again increases. However, as will be seen, this increase is more due to the lower signal to noise ratios associated with walkout than due to waves.

MGS aerobraking took place below $60^{\circ} \mathrm{N}$ so the polar vortex was not encountered, but MGS did show orbit to orbit variability of about $40 \% \quad 1-\sigma$ and a 
substantial fraction of this variability was due to stationary waves in the mid latitude region (Ref. 4). Explanation of such waves (Ref. 16) suggest that the stationary property is an artifact of sampling from a nearly inerially fixed orbit. The underlying waves are actually moving in the Mars atmosphere. Nevertheless, a large fraction of the orbit to orbit variability was modeled as stationary waves during MGS operations (Ref. 3). MGS persistence was found to follow a gamma probability distribution function (Ref. 4). The lower part of Fig. 14 provides the maximum likelihood estimate (MLE) of the gamma distribution that fits Odyssey persistence. The parameters " $a$ " and " $b$ " are such that "a" time " $b$ " is the mean and " $b$ " is the variance of the sample. With each estimate is shown the $95 \%$ confidence interval. For MGS (Ref. 4), $a=7.4(6.7-9.2)$ and $b=0.14(0.13-0.16)$. So even though the underlying physical process for the large variability seems to be different for the two missions, the statistical distribution of orbit to orbit variability is very similar. The Mars atmospheric model proposed for Odyssey Monte Carlo simulations (Ref. 4) gave gamma distribution parameters of $a=6.9(6.1-7.6)$ and $b=0.16(0.14-0.17)$ and provides an intermediate distribution that overlaps the distributions of the two missions.

Another simple approach is to scale the MarsGRAM density profile to have the same area under the curve as the accelerometer derived density profile. This essentially means the scaled MarsGRAM profile would have provided the same effective $\Delta \mathrm{V}$ as was measured by the accelerometers. Except for the small differences in drag coefficient models, this scaling provides a direct comparison with the NAV calculated $\Delta \mathrm{V}$ and an independent check on both approaches. Fig. 15 provides a comparison of these two methods in the form of the difference of the accelerometer derived $\Delta \mathrm{V}$ and the NAV

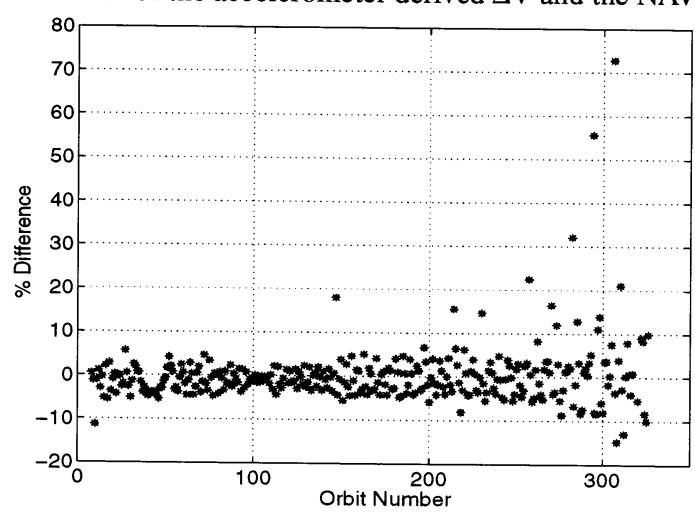

Fig. 15 Comparison of total drag $\Delta V$ from radio tracking and accelerometer data.

radio tracking $\Delta \mathrm{V}$ divided by the accelerometer value.
Over the entire mission, the mean difference is $0.12 \%$ with a RMS difference of $7 \%$. The large deviations during walkout are due to the total $\Delta \mathrm{V}$ becoming small, so signal to noise for both methods is increasing. While the drag is high and periapsis is inside the vortex, the agreement is within the uncertainty produced by the utilization of two $\mathrm{C}_{\mathrm{y}}$ models as discussed above.

\section{Stationary and Moving Waves}

During MGS aerobraking, longitudinal density waves were detected (Ref. 3). As mentioned earlier, the current explanation is that these waves are eastward moving waves on Mars that appear to be fixed in longitude because the observations are taken in an orbit with slow nodal precession. Before this insight was available, the waves were modeled as stationary waves relative to the rotating planet for MGS operations. The waves are identified with wave numbers, e.g. wave 1 goes through a complete sine cycle in $360^{\circ}$ of longitude, wave 2 goes through a complete cycle in $180^{\circ}$, etc. Waves 1 through wave 5 were detected at various times during the MGS mission (Ref. 13) and were used as a basis (Ref. 4) for preflight simulations using MarsGRAM (Ref. 14).

Based on the MGS experience, such stationary waves were expected for Odyssey and AMT procedures called for development of wave models on a daily basis. Unlike MGS, these models generally proved to have little predictive capability. It was soon found that assuming that the waves moved in longitude provided improved prediction. But again this capability was not sustained for extended periods of time. The wave modeling therefore proved to be of limited quantitative value during operations, but did provide qualitative knowledge on the state of the atmosphere that aided in making some maneuver decisions.

Post flight analysis of the data provided insight into the wave structure. The amplitude and phase of wave 1 are shown in Fig. 16. These results are based on daily least squares solutions for the mean density and only stationary wave 1 amplitude and phase. At least 12 orbits or 3 days of data, whichever is greater, is included in each solution. Up to day 32 there are 12 orbits in each solution and after that there are three days of data. The first solution encompasses 7 days and the last solution includes 36 orbits. Amplitude is given as a fraction of the mean density. In each solution set, the periapsis density (Fig. 12) is mapped, using the periapsis scale height, to the highest altitude in the set. Amplitude increases up through day 18 as periapsis approaches the pole, with the peak amplitude of $70 \%$ occurring when periapsis latitude is about $77^{\circ}$. Periapsis is north of $80^{\circ}$ from day 25 to day 43 and the amplitude is significantly lower, which might be considered as being inside the 
vortex. Periapsis is at $75^{\circ}$ and precessing south on day 47. Passing through the vortex during this time only produces an amplitude of $25 \%$. Outside the high latitude region the amplitude averages about $20 \%$.
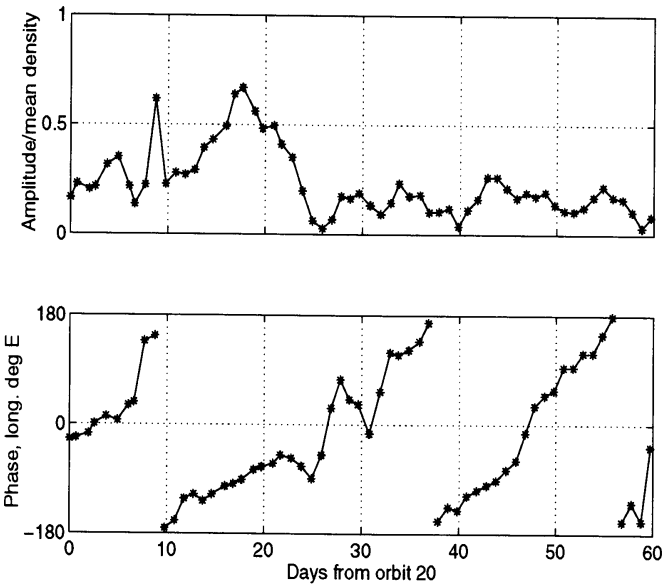

Fig. 16 Wave 1 amplitude and phase based on 12 orbits or 3 day fits to near periapsis density.

It is clear that wave 1 is generally progressing eastward throughout the entire aerobraking phase at an average rate of about $17^{\circ}$ day. MGS results for northern latitudes less than $60^{\circ}$ had average amplitudes of about $15 \%$ but the phase remained relatively constant (Ref. 4).

Results from a similar analysis for wave 2 are given in Fig. 17. Like wave 1, peak amplitude occurs prior to entering the vortex and decreases thereafter. This wave
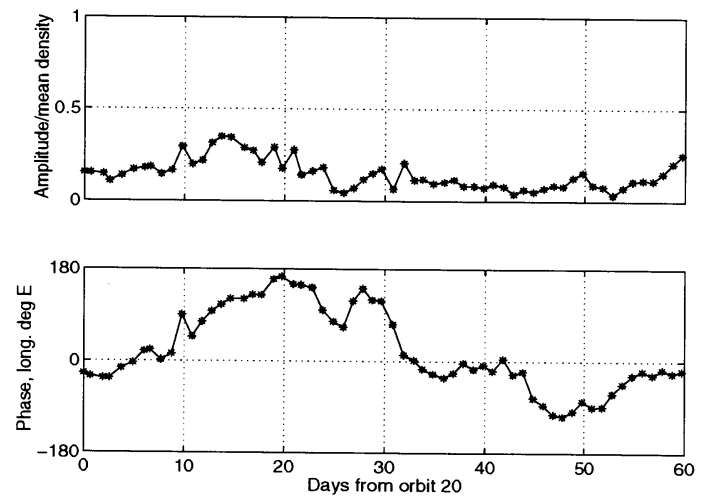

Fig. 17 Wave 2 amplitude and phase based on 12 orbits or 3 day fits to periapsis density.

also drifts eastward until the vortex is encountered then drifts westward until out of the vortex. The amplitude of about $10 \%$ for the last 15 days is consistent with MGS amplitudes in the $30^{\circ}$ to $60^{\circ}$ latitude range. However, during MGS the phase was relatively constant at about $50^{\circ}$ east. So even though there are some similarities with MGS, there are differences perhaps due to season, dust storm or local solar time differences.

\section{Mapping to Reference Altitude and Polar Plots}

Encountering the polar vortex required developing new analysis methods as the need arose. The persistent large waves that were seen on the inbound legs of the initial orbits suggested an increased risk as periapsis precessed toward the pole. It was of interest to quantify these waves in terms of the equivalent periapsis density variations. A number of approaches were attempted, but the best approach was to map the entire density profile to periapsis or some other altitude using a simple model of the atmosphere. The model used Eq. 3 and assumed that both the density and scale height at the reference altitude vary linearly with latitude or down track angle. Down track angle or true anomaly was used when periapsis was near the pole and latitude was not monotone over the pass. This approach presents a nonlinear optimization problem which was solved using an off the shelf constrained optimization routine. Convergence was robust but on occasions the solution was constrained by the upper and lower bounds.

A typical result is shown in Fig. 18. The upper figure shows the 7 second.mean density profile and the model
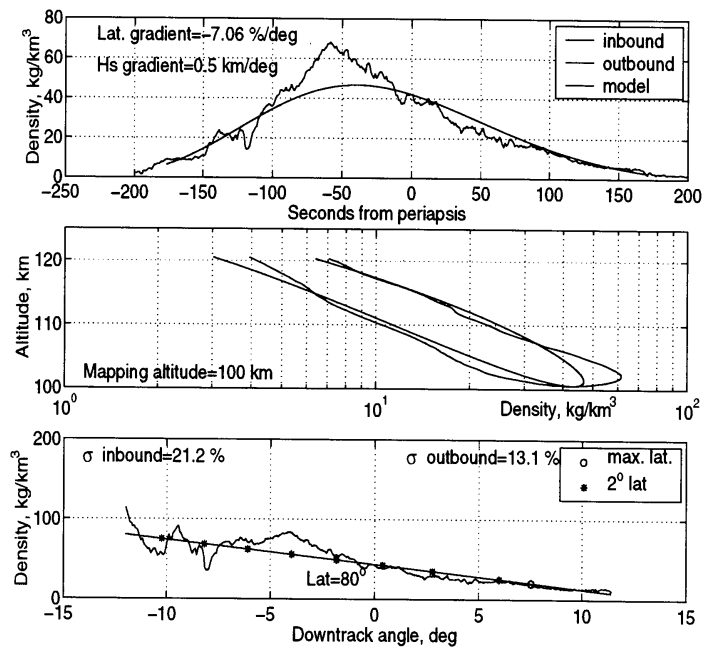

Fig. 18 Example of mapping to a reference altitude for wave detection, $\mathbf{P} 159$

fit to these data. The maximum densities differ significantly. The density latitudinal gradient is about $7 \%$ per degree down track and the scale height gradient is at the upper limit constraint of $0.5 \mathrm{~km}$ per degree down track. The scale height constraint corresponds to about $7 \%$ change in temperature per degree. The middle 
plot gives the altitudinal variation using the 39 point average density. Note that the density changes by a factor of three within three $\mathrm{km}$ of periapsis suggesting very strong latitudinal gradients or wave activity. The lower plot shows both the model and measured densities mapped to an altitude of $100 \mathrm{~km}$ using the model. The track is closest to the pole at about $7^{\circ}$ outbound. The red dots correspond to 2 degree latitude increments with periapsis occurring at about $82^{\circ}$. Apsidal regression is moving periapsis to the left in the lower figure, so one interpretation is that about $4^{\circ}$ ahead there is a region where the density is about twice as great at the same periapsis altitude. Also the RMS deviation from the model is $21.2 \%$ of the model density inbound and only $13.1 \%$ outbound. This again demonstrates that inside the vortex the variability is much less than outside the vortex. Like the terrestrial northern hemisphere jet stream, features like those shown in Fig. 18 are know to be highly variable in both space and time.

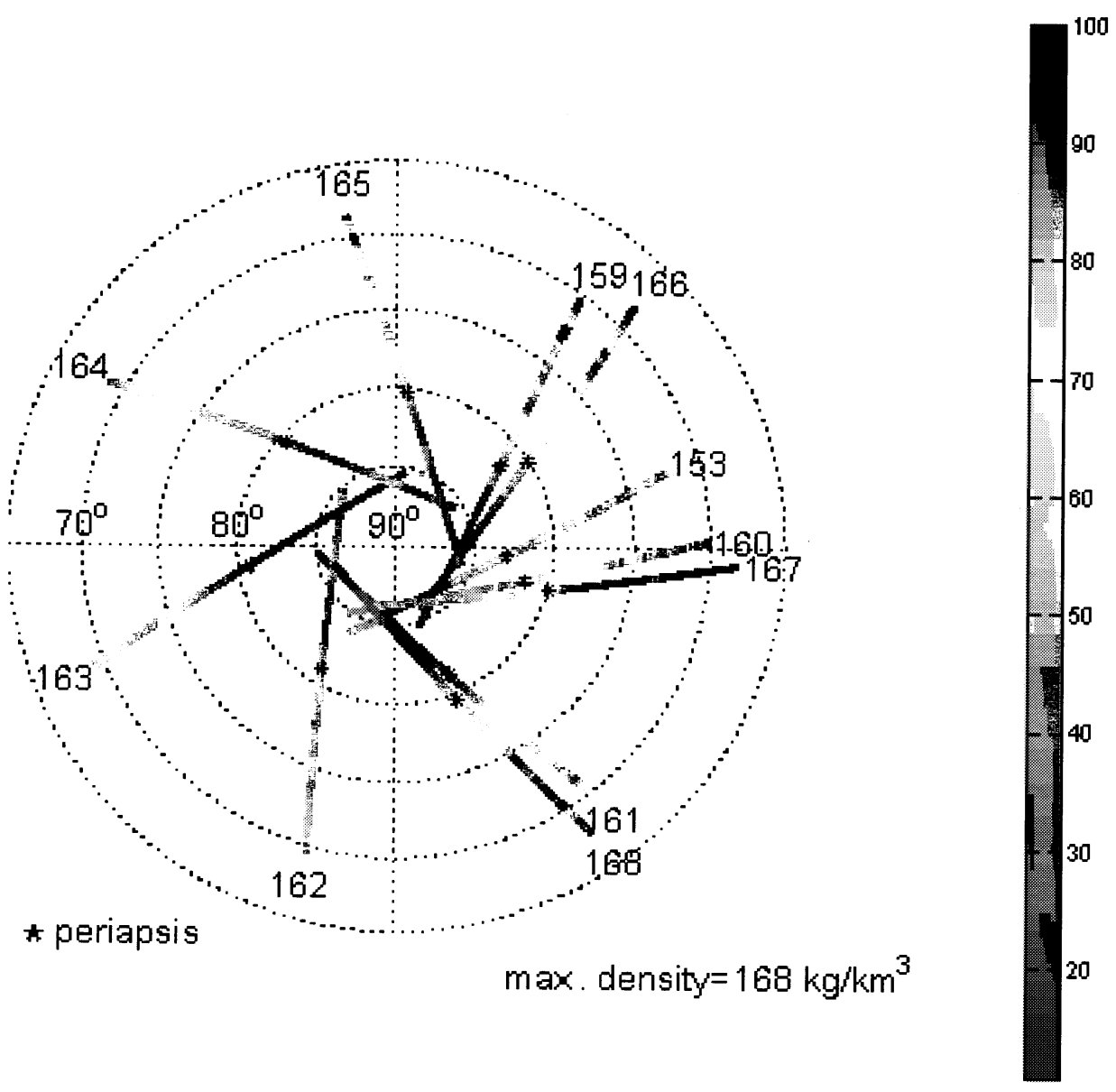

Fig. 19 Polar plot of densities mapped to $100 \mathrm{~km}$ altitude for orbits 153 and 159 through 168 . Density truncated at $100 \mathrm{~kg} / \mathrm{km}^{3}$ to enhance variability.

In an attempt to understand the temporal and spatial variations, polar plots of the density variation were made daily for orbits from the previous days. One such polar plot is shown in Fig. 19. Each track on the plot shows the density along the orbit path on a latitude- longitude polar plot. All of the measured densities have been mapped to a common reference altitude, in this case $100 \mathrm{~km}$. Each mapping is done using results similar to those in Fig. 18. The densities correspond to the data line in the lower subplot. The maximum predicted 
density of $168 \mathrm{~kg} / \mathrm{km}^{3}$ corresponds to the beginning of the inbound track for orbit 167 . To accentuate the along track variations, the color scale is truncated at $100 \mathrm{~kg}$ / $\mathrm{km}^{3}$ Periapsis is identified by a star on each track. The time between close orbit pairs (e.g. 160 and 167) is about 1 sol and such adjacent pairs were compared to generate confidence in the mapping and to identify trends in the movement of the density field. A strong wave 1 pattern is clearly evident at this time, with maximum density occurring in the first and fourth quadrants. Further, along track variability is small within $10^{\circ}$ of the pole and increases as latitude moves south. Along track variability is most apparent in orbits 159, 165, and 166. The strong latitudinal density gradient away from the polar region is also quite evident, particularly in the first and fourth quadrants. Plots like these were generated daily and provided considerable insight into the density morphology.

\section{Conclusions}

Aerobraking at Mars is both an opportunity and a challenge. Even if accurate predictive models were available, s/c design, mission design and operations would have to account for about a factor of two change in density from orbit to orbit or else be prepared to make periapsis trim maneuvers nearly every orbit. Accelerometer data complements radio tracking data in providing another means of obtaining the $\Delta \mathrm{V}$ due to drag on each pass. Further, these data provide information on the physical processes behind the variability, can be used to quantify the spatial and temporal variations, and will lead to improved Martian thermospheric models and understanding.

\section{Acknowledgement}

This work was sponsored by the NASA Mars ' 01 Odyssey project office and the NASA LaRC.

\section{References}

${ }^{1}$ Lyons, D. T., "Aerobraking Magellan: Plan versus Reality," Advances in the Astronautical Sciences, Vol. 87, Pt. 2, 1994, pp. 663-680.

${ }^{2}$ Lyons, D. T., et al., "Mars Global Surveyor: Aerobraking Mission Overview," Journal of Spacecraft and Rockets, Vol. 36, No 3, 1999, pp. 307-313.

3 Tolson, R. H., et al., "Application of Accelerometer Data to Mars Global Surveyor Aerobraking Operations," Journal of Spacecraft and Rockets, Vol 36, No 3, pp. 323-329, 1999.
4 Dwyer, A. M., et. al., "Development of a Monte Carlo Mars-GRAM Model for Mars 2001 Aerobraking Simulations" Advances in the Astronautical Sciences, Vol. 109, 2001, pp. 1293-1308.

${ }^{5}$ Tartabini, P, et al. "Development and Evaluation of an Operational Aerobraking Strategy for the Mars 2001 Odyssey," AIAA 2002-4975, Astrodynamics Specialist Conference, Monterey, CA, August 5, 2002.

${ }^{6}$ Dec, J., et al., "Thermal Analysis and Correlation of the Mars Odyssey Spacecraft's Solar Array During Aerobraking Operations", AIAA 2002-4536, Astrodynamics Specialist Conference, Monterey, CA, August 5, 2002.

7 Smith, J. "2001 Mars Odyssey Aerobraking” AIAA 2002-4532, Astrodynamics Specialist Conference, Monterey, CA, August 5, 2002

${ }^{8}$ Takashima, N. and Wilmoth, R. G., "Aerodynamics of Mars Odyssey," AIAA-2002-4809, 2002 Atmospheric Flight Mechanics Conference, Monterey, CA, August 5, 2002.

${ }^{9}$ Hanna, J. L. et. al., "Modeling Reaction Control System Effects on Mars Odyssey Aerodynamics," AIAA 2002- 4534, Astrodynamics Specialist Conference, Monterey, CA, August 5, 2002.

${ }^{10}$ Cestaro, F. J., and Tolson, R. H., "Magellan Aerodynamic Characteristics During the Termination Experiment Including Thruster Plume-Free Stream Interactions," NASA CR-1998-206940, March 1998.

11 Antreasian P., et. al., "2001 Mars Odyssey Orbit Determination During Interplanetary Cruise," AIAA 2002-4531, Astrodynamics Specialist Conference, Monterey, CA, August 5, 2002.

12 Mase, R., et. al., "The Mars Odyssey Navigation Experience," AIAA 2002-4530, Astrodynamics Specialist Conference, Monterey, CA, August 5, 2002.

${ }^{13}$ Tolson, R. H., et al., "Utilization of Mars Global Surveyor Accelerometer Data for Atmospheric Modeling," Astrodynamics 1999, Vol 103, American Astrodynamics Society, 1999.

14 Justus, C.G. and James, B.F., Mars Global Reference Atmospheric Model 2000 Version (Mars-GRAM 2000) Users Guide," NASA/TM-2000-21-279, May 2000.

15 Keating, G.M., et al., "Detection of winter polar warming in Mars upper atmosphere," Paper PS1.021TH2A-006, EGS XXVII General Assembly, Nice, France, April 2002

16 Wilson, J and Hamilton, K, "Comprehensive Model Simulation of Thermal Tides in the Martian Atmosphere," J. of Atmos. Sci., Vol 53, May 1, 1996, pp. 1290-1326. 